\title{
Molecular Detection of Human Herpes Viruses 1, 2, 3 and 6 in the Cerebrospinal Fluid of Patients with Central Nervous System Infections
}

\author{
Magda Salah El Dine Gabr, Nevine Nabil Kassem, Samia Abdou Girgis, Rania Mohamed Abd- \\ El Halim, Yasmeen Mohammad Mahmood Ali
}

Clinical Pathology Department - Faculty of medicine - Ain Shams University

\begin{abstract}
Background: Infections of central nervous system (CNS), such as encephalitis, meningitis, and meningoencephalitis are potentially life-threatening syndromes. They are caused by a diverse array of infectious causes. Despite being relatively uncommon, the morbidity, mortality, and costs are substantial. Of major importance are the herpes simplex virus (HSV)-2 and varicella zoster virus (VZV) in meningitis, and HSV-1 in encephalitis. Human herpes virus 6 (HHV-6) infection can induce severe encephalitis cases, particularly in immunocompromised. Human herpes viruses (HHVs) can cause CNS disease following primary infection, reactivation or recurrence.

Purpose: To estimate the frequency of four common neurotropic herpes viruses: HHV-1 (HSV-1), HHV-2 (HSV-2), HHV-3 (VZV) and HHV-6 as causative agents for viral encephalitis and aseptic meningitis.

Patients and Methods: The study was conducted on sixty five (65) CSF samples selected from the CSF of patients clinically diagnosed with acute encephalitis or meningitis. The samples belonged to 39(60.0\%) males and $26(40.0 \%)$ females. Their ages ranged from 12 days to 62 years old. All CSF samples included in the study were subjected to white cell count, estimation of the level of protein and glucose, direct macroscopic and microscopic examination and culture on blood, chocolate and MacConkey agar plates. All samples had a CSF leukocytic count $\geq 5$ cells/ $\mathrm{mm} 3$ and all were negative for bacterial culture. Real time PCR for the following viruses: HHV-1 (HSV-1), HHV-2 (HSV-2), HHV-3 (VZV) and HHV-6 was done for each sample.

Results: The results of the PCR showed that twenty six (40\%) were positive: twenty two (34\%) were positive for a single infection; HSV-1 was positive in seven (10.8\%), HSV-2 in eight (12.3\%), VZV in one (1.5\%) and HHV-6 in six $(9.2 \%)$ of the cases. Four (6.2\%) samples showed co-infection with two viruses. VZV was common in all cases of co-infection, together with HSV-2 in two (3\%), HSV-1 in one (1.5\%) and HHV-6 in one $(1.5 \%)$. Thirty nine cases $(60.0 \%)$ were negative for the four viruses.

Conclusion: The four herpes viruses (HSV-1, HSV-2, VZV and HHV-6) tested by PCR are causative agents in $40 \%$ of the tested cases of CNS infections. The most frequently detected virus by PCR was HSV-2 followed by HSV-1. In case of encephalitis, the most frequently detected virus was HSV-1, while HSV-2 was the most frequent in case of aseptic meningitis. VZV was common in all cases of co-infections.
\end{abstract}

Keywords: Meningitis, encephalitis, CNS infections, Herpes viruses, PCR.

\section{INTRODUCTION}

Infections of central nervous system (CNS), such as encephalitis, meningitis, and meningoencephalitis, remain a major global cause of morbidity and mortality ${ }^{(1)}$. In the United States, each year, there are approximately 7.3 hospitalizations per 100,000 population due to encephalitis. However, the syndromes encephalitis and meningitis are not nationally notifiable infectious conditions and therefore the incidence of these diseases is not well documented ${ }^{(\mathbf{1})}$.

Acute encephalitis is characterized by a triad of fever, headache, and altered mental status. Diffuse or focal neurologic signs such as cranial nerve dysfunction, hemiparesis, or seizures are commonly encountered. Capillary and endothelial inflammation of cortical vessels is a striking pathologic finding, occurring primarily in the gray matter or the graywhite junction. Aseptic meningitis refers to a disease with acute onset of symptoms and obvious signs of meningeal involvement, such as fever, headache, photophobia, stiff neck, irritability, nausea, vomiting, and rash. Negative bacterial culture of the cerebrospinal fluid (CSF) is suggestive of aseptic meningitis. Although the etiologic agent is not identified for most cases, viral infection has been reported as a major cause ${ }^{(2)}$.

Herpes simplex virus-1 (HSV-1) and varicella-zoster virus (VZV) are the first and second most common causes of viral encephalitis, respectively. These viruses are associated with mortality and morbidity, especially when treatment is delayed ${ }^{(3)}$. HSV-1 is responsible for approximately $10 \%$ of all cases of encephalitis and is the most common cause of fatal sporadic viral encephalitis worldwide. The mortality rate of HSV encephalitis (HSVE) may be more than 70\% if untreated, and more than $95 \%$ of untreated survivors will suffer lifelong sequelae. Varicella-zoster encephalitis affects 0.1 to $0.2 \%$ of persons with primary varicella. Before the use of varicella vaccine, it was the most common cause of 
encephalitis in children in Europe. In a recent UK study, it remains second only to HSV among infectious causes of encephalitis (5\%), and occurs mainly in the immunocompromised; $10 \%$ of whom were less than 20 years of age ${ }^{(4)}$. Infection with HSV-2 may result in meningitis or meningoencephalitis, which may recur despite therapy. Neonatal infection with HSV-2 is especially devastating, and disseminated disease may occur in approximately $25 \%$ of cases ${ }^{(5)}$. Human herpes virus-6 (HHV-6) has two distinct variants; HHV-6A and HHV-6B. Primary infection with HHV-6B in infants causes a non-specific febrile illness or most commonly exanthem subitum (roseola or 6th disease), and is associated with febrile convulsions. Up to $7 \%$ of children with encephalitis syndrome of otherwise unknown cause have HHV-6 DNA detectable in CSF with or without specific antibody responses ${ }^{(4)}$.

A distinction must be made between acute viral encephalitis (VE) and autoimmune/post-infectious encephalitis, which can occur with a variable latent phase between acute illness and the onset of neurologic symptoms. This distinction is critical because the management and prognosis are often quite different. Evaluation of cerebrospinal fluid (CSF) following lumbar puncture is essential for accurately diagnosing disease, unless its collection is contraindicated because of high intracranial pressure ${ }^{(2)}$.

Due to similarities in the clinical presentations of the diseases caused by these herpes viruses and other pathogens, virological testing is often needed ${ }^{(3)}$. The detection of herpes viruses in CSF using real-time PCR is now recognized as the gold-standard approach for diagnosing herpes encephalitis and herpes meningitis ${ }^{(5)}$. Studies suggest screening CSF cell count and protein values before performing HSV PCR in order to rationalize the use of this test. The only criteria proposed have been elevated CSF leukocyte counts $\left(>5\right.$ cells $/ \mathrm{mm}^{3}$ ) and/or protein levels (> $50 \mathrm{mg} / \mathrm{dl}){ }^{(6)}$.

\section{PATIENTS AND METHODS}

\section{Patients:}

The study included sixty five (65) CSF samples selected from the CSF of patients clinically diagnosed with acute encephalitis or meningitis and submitted to the Microbiology Laboratory at Ain Shams University Hospitals and Al-Abbassia Fever Hospital. The study was

\section{approved by the Ethics Board of Ain Shams University.}

Inclusion criteria: Patients diagnosed as having acute aseptic meningitis: Acute onset of meningeal symptoms i.e. headache, neck stiffness and/or photophobia without altered consciousness or change in behavior. CSF leukocytic count $\geq 5$ cells/ $\mathrm{mm}^{3}$. Patients diagnosed as having viral encephalitis if: They had evidence of brain parenchymal involvement without symptoms or signs of meningeal inflammation. Patients presenting with altered mental status (defined as decreased or altered level of consciousness, lethargy or personality change) lasting $\geq 24 \mathrm{~h}$ with no alternative cause identified. In addition to two or more of the following: Documented fever $\geq 38^{\circ} \mathrm{C}\left(100.4^{\circ} \mathrm{F}\right)$ within the $72 \mathrm{~h}$ before or after presentation. Generalized or partial seizures not fully attributable to a preexisting seizure disorder. New onset of focal neurologic findings. CSF leukocytic count $\geq 5$ cells $/ \mathrm{mm}^{3}$. Negative CSF bacterial culture.

Specimen collection and transport: Cerebrospinal fluid (CSF) samples were collected from patients with signs and symptoms of acute meningitis, encephalitis or meningoencephalitis via lumbar puncture under complete aseptic conditions into two separate $5 \mathrm{ml}$ sterile tubes for microbiology and chemistry. Specimens were then immediately transported to the laboratory for further processing.

Specimen processing: All CSF samples included in the study were subjected to: White Cell count. Estimation of the level of protein and glucose. Direct macroscopic and microscopic examination. Culture on blood, chocolate and MacConkey agar plates. Real time PCR for the following viruses: HHV-1 (HSV-1), HHV-2 (HSV2), HHV-3 (VZV) and HHV-6.

Real Time PCR: Real time PCR was performed on 65 CSF specimens with negative bacterial culture.

DNA extraction: Extraction of the viral DNA was done using QIAamp ${ }^{\circledR}$ MinElute ${ }^{\circledR}$ Virus Spin supplied by (Qiagen, USA) according to the manufacturer's instructions.

DNA amplification and detection: DNA amplification and detection was done by using QuantiTect ${ }^{\circledR}$ SYBR ${ }^{\circledR}$ Green PCR supplied by (Qiagen, USA), and using in-house designed primer sequences supplied by (Qiagen, USA), on Rotorgene Q real-time PCR thermal cycler (Qiagen, Germany). 
Forward Primers:

\begin{tabular}{|c|c|c|}
\hline HSV-1 & 5' GGC CTG GCT ATC CGG AGA 3' & \multirow{3}{*}{$\begin{array}{l}\text { (Càrdenas, } \\
\text { et al., 2014) }\end{array}$} \\
\hline HSV-2 & 5' AGA TAT CCT CTT TAT CAT CAG CAC CA 3' & \\
\hline VZV & 5' TCT TGT CGA GGA GGC TTC TG 3' & \\
\hline HHV-6 & 5'AAA ATT TCT CAC GCC GGT ATT C 3' & (1) \\
\hline
\end{tabular}

\section{Reverse Primers:}

\begin{tabular}{|l|l|l|}
\hline HSV-1 & 5' GCG CAG AGA CAT CGC GA 3' & \multirow{2}{*}{ (Càrdenas, et al., } \\
\cline { 1 - 2 } HSV-2 & 5' TTG TGC TGC CAA GGC GA 3' & \\
\hline VZV & 5' TGT GTG TCC ACC GGA TGA T 3' & \\
\hline HHV-6 & 5' CCT GCA GAC CGT TCG TCA A 3' & (1) \\
\hline
\end{tabular}

Principle:

Syber Green is a molecule that can be used in real-time PCR because it binds only to double-stranded DNA. When the PCR reaction occurs, a doublestranded copy is made and Syber Green binds and fluoresces when exposed to an excitation source ${ }^{(7)}$.

Detection takes place at the extension step of real-time PCR. Signal intensity increases with increasing cycle number due to the accumulation of PCR product. A major disadvantage for this dye is that it will detect the amplification of any DNA not just the gene of interest (may be contamination or primer dimer i.e. primers can sometimes anneal to themselves and create small templates for PCR amplification). Therefore, to be sure that the detected DNA is the target DNA, a melting curve should be done ${ }^{(8)}$.

Melting curve is done by thermal denaturation in which the products were heated after the final reaction from $60^{\circ} \mathrm{C}$ to $90^{\circ} \mathrm{C}$ and will produce a characteristic peak at the melting temperature of each amplicon (Tm: temperature at which $50 \%$ of the DNA is single stranded). A sudden decrease in fluorescence is detected when $\mathrm{Tm}$ is reached (due to dissociation of DNA strands and release of the dye). If only one amplicon is present, only one peak will be present. If primer dimers are present along with the product so a second peak (usually with reduced $\mathrm{Tm}$ because it is such a short DNA) will be seen ${ }^{(9)}$.

\section{Statistical Analysis:}

Data Management and Analysis: The collected data were revised, coded, tabulated and introduced to a PC using Statistical Package for Social Science (SPSS 20). Data were presented and suitable analysis was done according to the type of data obtained for each parameter.

Descriptive statistics: Median and Interquartile range (IQR) for non parametric numerical data. Frequency and percentage of non-numerical data.

Analytical statistics: Mann Whitney Test (U test) was used to assess the statistical significance of the difference of a non parametric variable between two study groups. Kruskal Wallis test was used to assess the statistical significance of the difference between more than two study group means. Post Hoc Test is used for comparisons of all possible pairs of group means Chi-Square test was used to examine the relationship between two qualitative variables Fisher's exact test: was used to examine the relationship between two qualitative variables when the expected count is less than 5 in more than $20 \%$ of cells

\section{RESULTS}

\section{Clinical and Laboratory Data:}

$39(60.0 \%)$ out of the 65 patients were males, while $26(40.0 \%)$ were females. In addition, $34(52.3 \%)$ were adults with age 18 years or older, while $31(47.7 \%)$ were pediatrics with age less than 18 years (table 1 ). The patient median age was 19 years, and the interquartile range (IQR) of their ages was (2-42) years.

Table (1): Patients' demographic data.

\begin{tabular}{|c|c|c|c|}
\hline & & $\mathbf{N}$ & $\%$ \\
\hline \multirow{2}{*}{ Sex } & Male & 39 & $60.0 \%$ \\
\hline & Female & 26 & $40.0 \%$ \\
\hline \multirow{2}{*}{ Age } & Pediatrics & 31 & $47.7 \%$ \\
\hline & Adult & 34 & $52.3 \%$ \\
\hline
\end{tabular}

Table (2) showed that: $31(47.7 \%)$ out of the 65 patients were clinically diagnosed with encephalitis, $30(46.2 \%)$ were diagnosed with meningitis, while $4(6.2 \%)$ were diagnosed with meningoencephalitis.

Table (2): Clinical diagnosis of CNS infections

\begin{tabular}{|l|l|c|c|}
\hline \multicolumn{2}{|c|}{} & $\mathbf{N}$ & \% \\
\hline \multirow{3}{*}{ Diagnosis } & Encephalitis & 31 & $47.7 \%$ \\
\cline { 2 - 4 } & Meningitis & 30 & $46.2 \%$ \\
\cline { 2 - 4 } & Meningoencephalitis & 4 & $6.2 \%$ \\
\hline
\end{tabular}

Table (3) showed that the CSF chemical analysis and cell count in our clinical cases. The CSF glucose level ranged between 10-116 mg/dL, with a median of $52 \mathrm{mg} / \mathrm{dL}$ and an IQR of 39-74 $\mathrm{mg} / \mathrm{dL}$. The CSF protein level ranged between 4$620 \mathrm{mg} / \mathrm{dL}$ with a median of $59 \mathrm{mg} / \mathrm{dL}$ and an IQR of $35-82 \mathrm{mg} / \mathrm{dL}$. The CSF WBC count ranged from $5-11326 / \mathrm{mm} 3$ with a median of $70 / \mathrm{mm} 3$ and an IQR of $15-210 / \mathrm{mm} 3$ as shown in table (3).

Table (3): CSF analysis of clinical cases.

\begin{tabular}{|l|c|c|}
\hline & Median (IQR) & Range \\
\hline Glucose (mg/dl) & $52(39-74)$ & $10-116$ \\
\hline Protein & $59(35-82)$ & $4-620$ \\
\hline CSF WBCs/mm3 & $70(15-210)$ & $5-11326$ \\
\hline
\end{tabular}

IQR: Interquartile range. CSF: Cerebrospinal fluid. WBCs: White blood cells 
In table (4) the macroscopic examination showed that $24(36.9 \%)$ samples were clear, 38 $(58.5 \%)$ were slightly turbid and $3(4.6 \%)$ were blood tinged. It also showed that $15(23.1 \%)$ had mild pleocytosis with CSF WBC count $5-10 / \mathrm{mm} 3,33$ (50.8\%) had moderate pleocytosis with CSF WBC count 11-200/mm3 and 17 (26.2\%) had marked pleocytosis with CSF WBC count more than $200 / \mathrm{mm} 3$. It also showed that the predominant type of cells was lymphocytes in $53(81.5 \%)$, neutrophils in $4(6.2 \%)$ and mixed in $8(12.3 \%)$ of the cases. All samples showed no pus and no organisms on Gram stain examination. They were also negative for acidfast bacilli on Ziehl-Neelsen stain examination.

Table (4): Macroscopic and microscopic examination.

\begin{tabular}{|c|c|c|c|}
\hline & & $\mathbf{N}$ & $\%$ \\
\hline \multirow{3}{*}{$\begin{array}{l}\text { Macroscopic } \\
\text { examination }\end{array}$} & Clear & 24 & $36.9 \%$ \\
\hline & Slightly turbid & 38 & $58.5 \%$ \\
\hline & Blood-tinged & 3 & $4.6 \%$ \\
\hline \multirow{3}{*}{ Pleocytosis } & Mild & 15 & $23.1 \%$ \\
\hline & Moderate & 33 & $50.8 \%$ \\
\hline & Marked & 17 & $26.2 \%$ \\
\hline \multirow{3}{*}{$\begin{array}{l}\text { Predominant cell } \\
\text { (Leishman stain) }\end{array}$} & Lymphocyte & 53 & $81.5 \%$ \\
\hline & Neutrophils & 4 & $6.2 \%$ \\
\hline & Mixed & 8 & $12.3 \%$ \\
\hline
\end{tabular}

Table (5) showed that the results of the PCR for the detection of the four tested viruses (HSV-1, HSV-2, VZV and HHV-6). Out of 65 negative bacterial culture: $39(60.0 \%)$ where negative for the four viruses and $26(40 \%)$ showed positive results.

Out of the 26 positive PCR results: 22 $(84.6 \%)$ were positive for a single infection and four $(15.4 \%)$ showed viral co-infection.

The 22 with single viral infection were seven HSV-1, eight HSV-2, one VZV and six HHV-6.

The four CSF samples showing coinfection: One (VZV+HSV-1), Two (VZV+HSV-2) and one (VZV+HHV-6).

Table (5): PCR results of the 65 cases of CNS infections

\begin{tabular}{|l|c|c|c|}
\hline & $\mathbf{N}$ & $\begin{array}{c}\text { \% of total } \\
(\mathbf{6 5})\end{array}$ & $\begin{array}{c}\text { \% of positive } \\
(\mathbf{2 6})\end{array}$ \\
\hline Negative & 39 & $60.0 \%$ & \\
\hline Single infections & 22 & $33.8 \%$ & $84.6 \%$ \\
\hline HSV1 & 7 & $10.8 \%$ & $26.9 \%$ \\
\hline HSV2 & 8 & $12.3 \%$ & $30.8 \%$ \\
\hline VZV & 1 & $1.5 \%$ & $3.8 \%$ \\
\hline HHV6 & 6 & $9.2 \%$ & $23.1 \%$ \\
\hline Co-infection & 4 & $6.2 \%$ & $15.4 \%$ \\
\hline VZV HSV-1 & 1 & $1.5 \%$ & $3.8 \%$ \\
\hline VZV HSV-2 & 2 & $3 \%$ & $7.7 \%$ \\
\hline VZV HHV-6 & 1 & $1.5 \%$ & $3.8 \%$ \\
\hline
\end{tabular}

Table (6) showed the relationship between samples positive and negative results for the four viruses collectively as regard sex, age group, degree of pleocytosis, predominant type of cells and clinical diagnosis. There was no statistically significant difference between the samples that are positive for any of the four viruses (22 single and 4 coinfections) and those that were negative for the same viruses in relation to CNS infections (encephalitis, meningitis and meningoencephalitis) with a p-value $>0.05$, although almost half of the cases were due to the viruses detected. There was also no statistically significant relation between positive cases for any of the four viruses and either of the age, sex, pleocytosis or predominant type of cells with a p-value $>0.05$. The IQR of the age of positive cases was 2 - 48 years, with a median of 25 years.

Table (6): Collective results of the four viruses as regard clinical and CSF data.

\begin{tabular}{|c|c|c|c|c|c|c|c|}
\hline & \multicolumn{4}{|c|}{ Virus } & \multirow{2}{*}{\multicolumn{2}{|c|}{ Chi square test }} \\
\hline & & \multicolumn{2}{|c|}{ Negative } & \multicolumn{2}{|c|}{ Positive } & & \\
\hline & & $\mathbf{N}$ & $\%$ & $\mathbf{N}$ & $\%$ & p value & sig. \\
\hline \multirow{2}{*}{ Sex } & Male & 24 & $61.5 \%$ & 15 & $57.7 \%$ & \multirow{2}{*}{0.756} & \multirow{2}{*}{ NS } \\
\hline & Female & 15 & $38.5 \%$ & 11 & $42.3 \%$ & & \\
\hline \multirow{2}{*}{ Age } & Pediatrics & 21 & $53.8 \%$ & 10 & $38.5 \%$ & \multirow{2}{*}{0.224} & \multirow{2}{*}{ NS } \\
\hline & Adult & 18 & $46.2 \%$ & 16 & $61.5 \%$ & & \\
\hline \multirow{3}{*}{ Pleocytosis } & Mild & 10 & $25.6 \%$ & 5 & $19.2 \%$ & \multirow{3}{*}{0.832} & \multirow{3}{*}{ NS } \\
\hline & Moderate & 19 & $48.7 \%$ & 14 & $53.8 \%$ & & \\
\hline & Marked & 10 & $25.6 \%$ & 7 & $26.9 \%$ & & \\
\hline \multirow{3}{*}{$\begin{array}{l}\text { Predominant } \\
\text { cell }\end{array}$} & Lymphocyte & 32 & $82.1 \%$ & 21 & $80.8 \%$ & \multirow{3}{*}{0.127} & \multirow{3}{*}{ NS } \\
\hline & Neutrophils & 4 & $10.3 \%$ & 0 & $0.0 \%$ & & \\
\hline & Mixed & 3 & $7.7 \%$ & 5 & $19.2 \%$ & & \\
\hline \multirow{3}{*}{ Diagnosis } & Encephalitis & 15 & $38.5 \%$ & 16 & $61.5 \%$ & \multirow{3}{*}{0.185} & \multirow{3}{*}{ NS } \\
\hline & Meningitis & 21 & $53.8 \%$ & 9 & $34.6 \%$ & & \\
\hline & Meningoencephalitis & 3 & $7.7 \%$ & 1 & $3.8 \%$ & & \\
\hline
\end{tabular}

NS: Non-significant

Table (7) showed the relationship between samples positive and negative results for the four viruses collectively, as regard CSF WBC count, CSF glucose and protein levels. There was no statistically significant difference between the samples that were positive for any of the four viruses and negative samples in relation to either of CSF WBC count, CSF glucose level or CSF protein level with a p-value $>0.05$.

Table (7): Collective results of the four viruses as regard CSF analysis:

\begin{tabular}{|c|c|c|c|c|c|c|}
\hline & \multicolumn{4}{|c|}{ Virus } & \multirow{2}{*}{\multicolumn{2}{|c|}{ Mann Whitney test }} \\
\hline & \multicolumn{2}{|c|}{ negative } & \multicolumn{2}{|c|}{ positive } & & \\
\hline & Median & IQR & Median & IQR & p value & sig. \\
\hline $\begin{array}{l}\text { CSF } \\
\text { WBCs } / \mathrm{mm}^{3}\end{array}$ & 95.0 & $10-240$ & 60.0 & $18-210$ & 0.936 & NS \\
\hline $\begin{array}{l}\text { Glucose } \\
(\mathrm{mg} / \mathrm{dl})\end{array}$ & 49.0 & $35-67$ & 58.0 & $48-80$ & 0.105 & NS \\
\hline Protein & 48.0 & $30-89$ & 61.0 & $47-74$ & 0.338 & NS \\
\hline
\end{tabular}

NS: Non-significant 


\section{DISCUSSION}

Viral infections of the CNS are not uncommon in clinical practice; the prevalence of these cases is not well defined. Whereas, the etiology of many cases remains unknown, an increasing number of positive viral identifications have been made, particularly since the advent of molecular diagnostics. Though specific therapies are unfortunately only available for a few viruses (e.g., herpes viruses and the human immunodeficiency virus), efforts to find causative organisms remain important for the effective management and prognosis of patients. Clinical and laboratory features common to particular viral etiologies can guide clinicians in identifying potential pathogens ${ }^{(\mathbf{1 0})}$.

Most HHV are neurotropic and a common cause for serious, acute, and chronic neurological disease of the CNS. The spectrum of clinical manifestations and disease outcomes are dependent on the specific pathogen and the immunologic state of the host. Improvement in molecular diagnostic method using PCR for viral DNA in CSF samples has enabled the study of the etiology of HSV-1, HSV-2, VZV and HHV-6 in CNS infection ${ }^{(11)}$.

In our study, 39/65 (60.0\%) of the patients were males, while $26 / 65(40.0 \%)$ were females. Adults were $34 / 65(52.3 \%)$ whose age was 18 years or older, while $31 / 65(47.7 \%$ ) were pediatrics with age less than 18 years old. The patients' ages ranged from 12 days to 62 years. Their median age was 19 years old, and the interquartile range (IQR) of their ages was (2-42) years old.

Similarly, a study was performed in Georgia by Akhvlediani and colleagues ${ }^{(12)}$. It showed that Over $51 \%$ of participants (134) were males. The mean age was 23.9 years. However, $58 \%$ of patients were children less than 18 years of age.

In addition, a study done in the USA by Dupuis and coworkers ${ }^{(1)}$ showed that $53 \%$ of the 2,357 participants were males. Twenty six percent of patients whose age was reported were under 20 years old; $74 \%$ were over 20 years old. In another study carried out in Brazil by Rimério and colleagues ${ }^{(10)}$, the patients' mean age was $23(0.1$ 74.4 years old). Males were 25/52 (48\%).

In our study, 31/65 (47.7\%) of the patients were clinically diagnosed with encephalitis, 30/65 (46.2\%) were diagnosed with meningitis, while $4 / 65$ (6.2\%) were diagnosed with meningoencephalitis.
A study done in the USA by Wootton and coworkers ${ }^{(13)}$, was concordant with our study, where 23/48 (47.9\%) were diagnosed with encephalitis and 25/48 (52\%) were diagnosed with meningitis at discharge.

Another study performed in South Korea by Choi and colleagues (11), showed that 41/135 patients were identified as having encephalitis, 70/135 (51.9\%) aseptic meningitis, and 24/135 (17.8\%) meningoencephalitis at discharge.

Rimério and coworkers ${ }^{(10)}$ reported that $22 / 52(42.3 \%)$ of cases were encephalitis, $3 / 52$ (5.8\%) of cases were meningitis and 3/52 (5.8\%) of cases were meningoencephalitis.

In Akhvlediani and associates ${ }^{(12)}$ study, 39/134 (29\%) patients were classified by treating clinicians as viral meningitis. Encephalitis accounted for $9 \%(12 / 134)$.

In the present work, detection of the four viruses (HSV-1, HSV-2, VZV and HHV-6) by real time PCR revealed that the total number of positive samples was $26(40 \%)$, while $39(60.0 \%)$ of the samples where negative for the four viruses. The HSV-1 was positive in 7 (10.8\% of 65 total and $26.9 \%$ of 26 positive of the total cases. The HSV-2 was positive in $8(12.3 \%, 30.8 \%)$ of the cases. The VZV was positive in $1(1.5 \%, 3.9 \%)$ of the cases. The HHV-6 was positive in $6(9.2 \%, 23 \%)$ of the cases. Four $(6.2 \%, 15.4 \%)$ samples showed coinfection with 2 viruses [ 2 cases $(3 \%, 7.7 \%)$ positive for VZV plus HSV-2, 1 case $(1.5 \%, 3.8 \%)$ positive for VZV plus HSV-1 and 1 case $(1.5 \%$, $3.8 \%$ ) positive for VZV plus HHV-6].

In the study performed by Rimério and colleagues ${ }^{(10)}$ in Brazil: DNA samples extracted from the CSF of neurological patients were positive in $27 / 52(51.9 \%)$ for all eight $\mathrm{HHV}$ infection using nested PCR. Unique HHV-DNA strand was detected in 10/27 (37\%) samples. Detection of two or more HHV-DNA occurred in 17/27 (63\%). Among the positive tests, the frequency of virus found in CSF samples was $37 \%$ for HHV-6, $18.5 \%$ for HSV-1, $11.1 \%$ for VZV, $7.4 \%$ for HSV-2, $55.6 \%$ for HCMV, $44.4 \%$ for EBV and $33.3 \%$ for HHV-7.

Wootton and coworkers ${ }^{(13)}$ identified pathogens in $14(29.2 \%)$ of 48 samples by PCR. Viral pathogens were most commonly detected [EBV (8), HSV2 (3), VZV (3), HSV1 (1), Enterovirus (1)], followed by bacterial and fungal. 
Viral co-detections were present in four patients $(8.3 \%)$; EBV was present in all (4) along with VZV (2), HSV1 (1), HSV2 (1).

According to the study done in South Korea by Choi and associates ${ }^{(11)}$, a total of 24 (17.8\%) out of 135 patients were diagnosed, using real time PCR, with one of the following three herpes viruses (HSV-1, HSV-2 or VZV) as causative agents of aseptic meningitis, meningoencephalitis or encephalitis. Among the 24 positive cases, 18 ( $75 \%$ of positives, $8.0 \%$ of total) patients with VZV, $4(16.7 \%, 1.7 \%)$ patients with HSV-2, and $2(8.3 \%, 0.9 \%)$ patients with HSV-1 were identified. There were no patients with mixed infections.

de Ory and colleagues (14) in Spain, assayed CSF samples by real time and multiplex PCR for enterovirus (EV), herpes virus (herpes simplex [HSV], varicella-zoster [VZV], cytomegalovirus [CMV], Epstein-Barr [EBV], and human herpes virus-6 [HHV-6]), mumps (MV), toscana virus (TOSV), adenovirus (AdV), lymphocytic choriomeningitis virus (LCMV), West Nile virus (WNV), and rabies. They reported 250 positive cases out of the 566 samples studied, using PCR (44.2\%): 31 were positive for HSV-1 (12.4\% of positives and $5.5 \%$ of the total); 28 for VZV (11.2\% and 4.9\%); three for HSV-2 (1.2\% and $0.5 \%)$; one HSV-non typed ( $0.4 \%$ and $0.2 \%)$; two HHV-6 (0.8\% and $0.4 \%)$.

In USA, Dupuis and coworkers (1) identified one or more viral agents, using real time and multiplex PCR, in 340 patient samples resulting in an overall detection rate of $14.4 \%$. Nine patients $(0.4 \%$ of total $)$ were positive for HSV-non typed. HSV-1 was detected in $22(0.9 \%)$ patient samples and HSV-2 was detected in 36 $(1.5 \%)$ patient samples. One patient had a dual infection with HSV-1 and -2 . The virus most detected after enterovirus, EBV, and HSV was VZV $44(1.9 \%)$. A total of $21(0.9 \%)$ HHV-6 positive specimens were detected. 22 dual infections were detected. These were mainly dual infections with EBV or HHV-6.

In our study, by comparing samples positive for each virus and coinfections with negative samples, there was a statistically significant difference in relation to CNS infections (encephalitis, meningitis and meningoencephalitis) with a p-value $<0.05$. However, there was no statistically significant relation with the age group, sex, pleocytosis or predominant type of cells with a $\mathrm{p}$-value $>0.05$. There is also no statistically significant relation with either of the age, CSF WBC count, CSF glucose level or CSF protein level with a p-value $>0.05$.

In our study, there was no statistically significant difference between the group of samples that was positive for any of the four viruses $(22$ single and 4 coinfections) and the group that was negative for the same viruses in relation to CNS infections (encephalitis, meningitis and meningoencephalitis) with a p-value $>0.05$. There was also no statistically significant relation between positive cases for any of the four viruses and either of the age group, age in years, sex, pleocytosis, predominant type of cells, CSF WBC count, CSF glucose level or CSF protein level with a $\mathrm{p}$-value $>0.05$. This could be explained by the possible presence of other viruses that were not detected in our study.

Similarly, the study done in Iran by Hosseininasab and associates ${ }^{(15)}$ on 65 children with meningitis. The CSF samples were tested for HSV and HHV-6 using real-time PCR and VZV using nested PCR, among other neurotropic viruses. It showed that there was no significant difference between the children with PCR positive and negative results with regard to age, gender and CSF profiles.

In a study done by Salah-Eldin and colleagues ${ }^{(16)}$ in Qena governorate. This study was conducted on 50 children with viral encephalitis. Their CSF samples were tested for HSV-1 and HSV-2 using real-time PCR. In accordance with our study, age had no significant effect on the result of PCR. However, in contrast to our study, female patients showed significant high frequency of positive PCR (81\%) in comparison to male patients (37.9\%). Regarding CSF findings, patients with HSV encephalitis typically had moderate elevation of CSF opening pressure, a moderate CSF pleocytosis, a mildly elevated CSF protein and normal CSF to plasma glucose ratio.

In the study done by Choi and colleagues ${ }^{(11)}$ and in contrast to our study median CSF WBC count was significantly higher in patients infected with HSV-2 (620 cells $/ \mathrm{mm} 3$ ) than in patients infected with HSV-1 $(90$ cells $/ \mathrm{mm} 3)$ or VZV $(210$ cells $/ \mathrm{mm} 3)(\mathrm{P}<0.05)$. However, in accordance with our study, no significant 
differences were found between median CSF WBC counts for HSV-1 and VZV (P>0.05). Other CSF findings in their study were not significantly different among the three groups of patients infected with each herpes virus or among the three groups classified as meningitis, encephalitis, or meningoencephalitis.

\section{CONCLUSION}

The four herpes viruses (HSV-1, HSV-2, VZV and HHV-6) tested by PCR were the causative agents in $40 \%$ of the studied cases of CNS infections. The most frequently detected virus by PCR was HSV-2 followed by HSV-1. Detection of VZV by PCR was common in all cases of co-infections.

\section{REFERENCES}

1. Dupuis M, Hull R, Wang $H$ et al. (2011): Molecular Detection of Viral Causes of Encephalitis and Meningitis in New York State. J. Med. Virol., 83: 2172-2181

2. Kelly TA, O'Lorcain $\mathrm{P}$, Moran $\mathrm{J}$ et al. (2013): Underreporting of Viral Encephalitis and Viral Meningitis, Ireland, 2005-2008. Emerg. Infect. Dis., 19 (9): 1428-1436

3. Pillet S, Verhoeven PO, Epercieux A et al. (2015): Development and Validation of a Laboratory-Developed Multiplex Real-Time PCR Assay on the BD Max System for Detection of Herpes Simplex Virus and Varicella-Zoster Virus DNA in Various Clinical Specimens. J Clin Microbiol., 53:1921-1926

4. Britton P, Jones C (2013): Central nervous system herpesvirus infections. Paed. Child Health, 24:6 248-254

5. Binnicker MJ, Espy MJ, Irish CL (2014): Rapid and Direct Detection of Herpes Simplex Virus in Cerebrospinal Fluid by Use of a Commercial Real-Time PCR Assay. J. Clin. Microbiol., 52(12): 4361-4362

6. Roa PL, Alonso R, Egea V et al. (2013): PCR for Detection of Herpes Simplex Virus in Cerebrospinal Fluid: Alternative Acceptance Criteria for Diagnostic Workup, J. Clin. Microbiol., 51(9):2880-2883

7. Muldrew KL (2009): Molecular diagnostics of infectious diseases. Curr. Opin. Ped., 21:102111

8. Soheili Z, Samiei $\mathbf{S}$ (2005): Real Time PCR: Principles and Application. Hepat Mon., 5(3): 83-87
9. Schmittgen, TD (2006): Quantitative gene expression by real-time PCR: a complete protocol. In: Real-time PCR. Dorak MT, ed. (BIOS Advanced Methods). New York, USA: Taylor and Francis.

10. Rimério CAT, De Oliveira RS, Bonatelli MQ et al. (2015): Human Herpesvirus Infections of the Central Nervous System: Laboratory Diagnosis Based on DNA Detection by Nested PCR in Plasma and Cerebrospinal Fluid Samples. J. Med. Virol., 87:648-655

11. Choi R, Kim GM, Jo IJ et al. (2014): Incidence and Clinical Features of Herpes Simplex Viruses (1 and 2) and Varicella-Zoster Virus Infections in an Adult Korean Population with Aseptic Meningitis or Encephalitis. J. Med. Virol., 86:957-962

12. Akhvlediani T, Bautista CT, Shakarishvili R et al. (2014): Etiologic Agents of Central Nervous System Infections among Febrile Hospitalized Patients in the Country of Georgia. PLoS ONE, 9(11): e111393

13. Wootton SH, Aguilera E, Salazar L et al. (2016): Enhancing pathogen identification in patients with meningitis and a negative Gram stain using the BioFire FilmArray ${ }^{\circledR}$ Meningitis/Encephalitis panel. Ann Clin Microbiol Antimicrob., 15:26

14. de Ory F, Avellón A, Echevarría JE et al. (2013): Viral Infections of the Central Nervous System in Spain: A Prospective Study. J. Med. Virol., 85:554-562

15. Hosseininasab A, Alborzi A, Ziyaeyan M et al. (2011): Viral Etiology of Aseptic Meningitis Among Children in Southern Iran. J.Med.Virol., 83:884-888.

16. Salah-Eldin EM, Aly SS, Mohey-El-Deen ZM et al. (2017): Clinical and Laboratory Characteristics of Herpes Simplex Encephalitis in Children in Qena Governorate, Egypt. Int $\mathbf{J}$ Clin Pediatr., 6 (1-2):1-7 